\title{
SYSTEMATIC VISION WITH HAND GESTURE RECOGNITION CONTROL BASED ON SECURED FUSION OF MOMENTS
}

\author{
Amandeep Walia ${ }^{1}$ \\ ${ }^{1}$ Automation \& Robotics, Ambalika Institute of Management \& Technology, Lucknow
}

\begin{abstract}
This work is focussed on three main issues in developing a gesture recognition system. These are (i) Threshold independent skin colour segmentation using Modified K-means clustering and Mahalanobish distance (ii) illumination normalization(iii) user independent gesture recognition based on fusion of Moments.
\end{abstract}

Keywords - American Sign Language, Human computer interaction, ROBOT Control ***.

\section{INTRODUCTION}

We mainly communicate with others with the help of our voice and body language. Although speech is the mostly used way of interaction for human beings, body language and facial expressions are also used to interact with others. In many cases, interaction with the physical world by body language and gestures is more reliable. Gestures or body languages can be expressed in various ways. These can be expressed by simply waving hands, making a meaningful gesture by hand, finger or body pose or by a meaningful facial expression. In between these gestures and expressions, hand gestures are the most efficient means to express meaningful and significant information. In our real life situation, we use hand gestures to communicate with mute and deaf people by using sign languages, to count numbers and to express a feeling like 'good bye' or 'stop'. With the recent developments in Artificial intelligent, Soft Computing and Neural Networks, hand gestures are becoming the most important tool to interact with computers and machines.

Generally, gestures are of two types: Static gestures and Dynamic gestures. Static gestures are mainly expressed by some meaningful body expressions. Simple 'Stop' gesture is a static hand gesture to express a significant information. Many times static gestures are used to express dynamic gestures also. In American Sign Language, digits greater than nine have been expressed by the hand movement and with a static gesture from zero to nine. Dynamic gestures are expressed by body movements. Simply waving hand to express 'good bye' is a dynamic gesture. Compared to other body parts, hands are more flexible. For that reason, hand gestures are mostly used in human computer interaction than other body parts.

\section{GESTURES}

We are interested in recognizing American Sign Language (ASL) [2] human hand gestures using computer vision principles. Gestures are usually understood as hand and body movement that can convey information and can be a proper means of communication between two persons. According to Webster's dictionary
A gesture is a pose or movement of some organs like hand, finger etc to convey certain kind of meaningful information. It is one of the important medium to communicate with others. Gestures are divided into two categories: I) Static gesture II) Dynamic gesture

A dynamic gesture is just movement of hand or any other body part over a period of time whereas a static gesture is a pose or position of hand or any other body part. Example of dynamic gesture may be just waving a goodbye and example of static gesture may be the stop sign. Some complex algorithms and methods are designed to understand and interpret various types of gestures over a period of time. This complex processes are called gesture .

\subsection{Gesture Based Applications}

Static and dynamic hand gestures have a huge application in both multidirectional control and sign language purpose.

Robot Control in Inaccessible Remote Areas: It is impossible for human beings to operate and physically present in a hostile condition like nuclear power plant, defence research plants, medicine manufacturing plants etc. In that case, a robot and gesture based robot control systems have a great use .

Virtual Reality: It is a computer aided environment which is analogous to real world. It is designed by a high quality of animation technology. It can be displayed through computer screen as well as special stereoscopic displays.

Sign Language: In sign languages with some movements and poses of body parts we communicate with mute and deaf people. It can be expressed by hand poses, hand gestures, movement of hands etc. Sign language recognition is one of the mostly used human computer interaction (HCI) application .

Remote Control : Static hand gesture recognition systems are on the run to replace the remote control devices of electronic gadgets. It is more reliable to control electronic gadgets by meaningful hand gestures than remote control devices. 
Automobile Control: Automobile industry is also in the run to use gesture based control of automobiles and its various accessories. Researchers are designing some gesture recognition systems for blind-spot recognition and parking assist. Some researchers are designing some automobiles which can be driven by using some gestures.

\section{DATABSE DESCRIPTION}

In this project all operations are performed on RGB colour images .We have made two hand gesture databases. In case of first database, a uniform black background is used behind the user to avoid background noises. Second one is taken in complex background. In second database hand region is restricted to have maximum area compared to other regions.

A Logitech c120 webcam has been used to capture the hand gesture images. The resolution of grabbed image is $320 \times$ 240 for both the databases. All images are taken in various angles and in different light conditions to make our gesture recognition algorithm rotation and illumination invariant. We have used the uniform background database only for semi-supervised learning purpose. Second database is mainly used for testing and training purpose. The dataset consists of 1500 gestures of 10 classes, 15 samples each class of 10 users. The dataset is equally divided into training and testing datasets of 750 gestures of 10 classes for 5 different users to make the system user-independent.

Two databases are given below

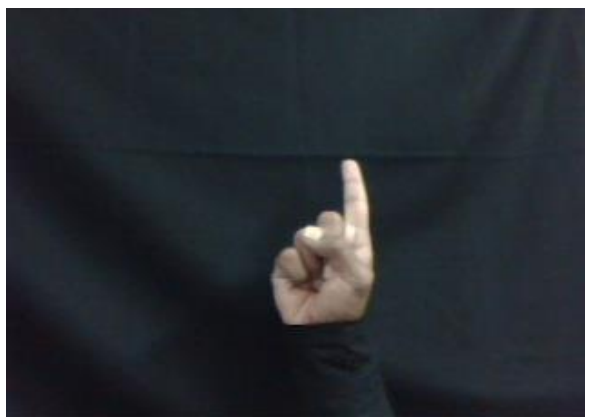

ASL Digit 1

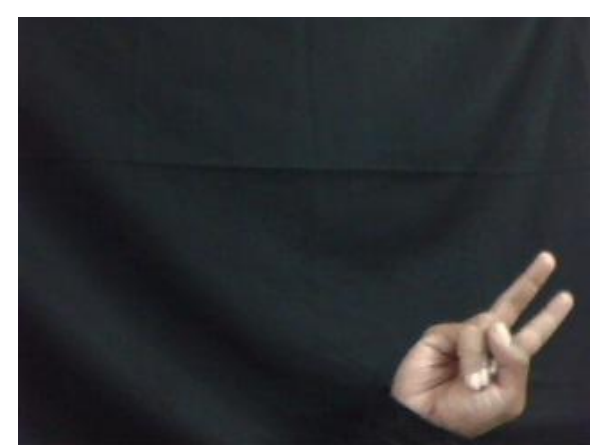

ASL Digit 2

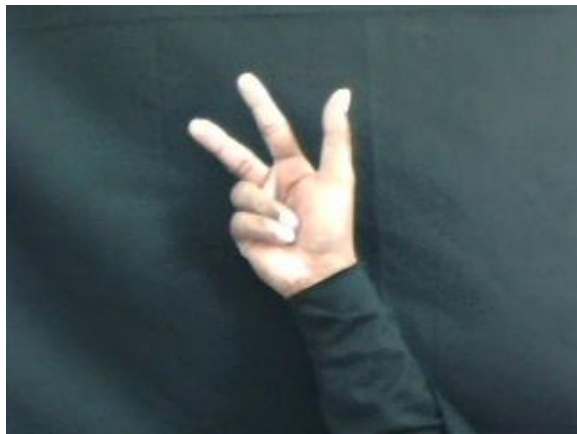

ASL Digit 3

Fig1 Sample image from Database 1

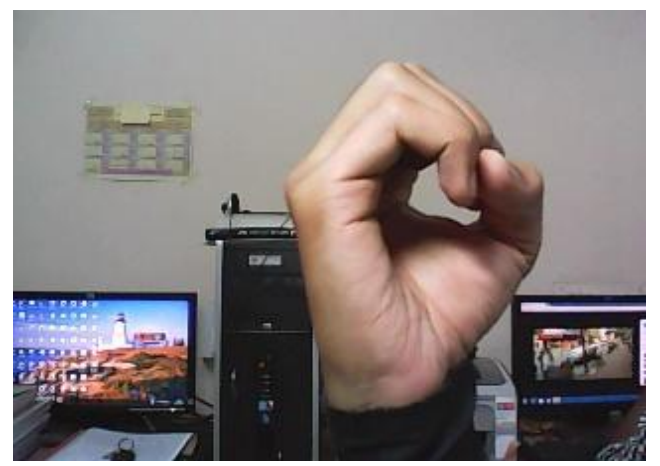

ASL Digit 0

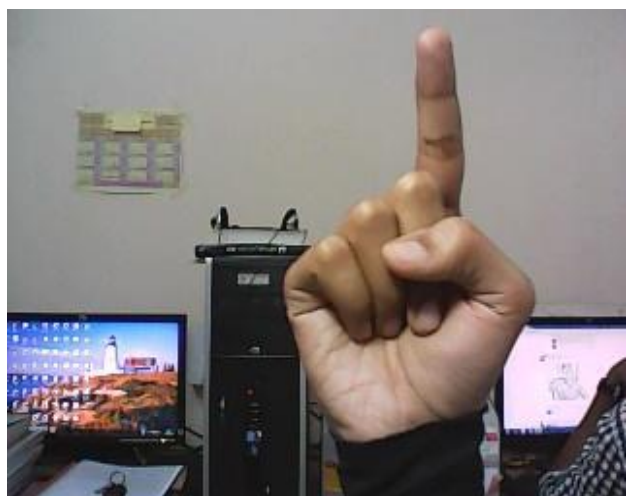

ASL Digit 1

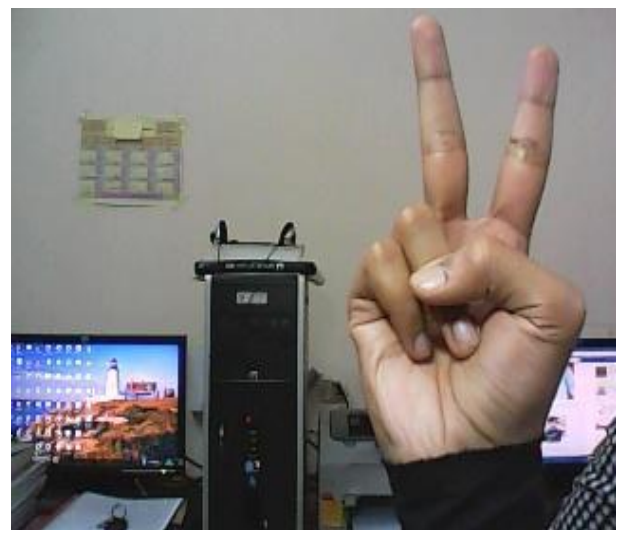

ASL Digit 2

Fig2 Sample images from Database 2 
In Static hand gesture recognition, pre-processing is the primary and the most important step. In pre-processing, binary silhouette of the hand gesture image is extracted for shape based feature extraction. For contour based feature extraction, boundaries are extracted from the colour hand gesture image. Pre-processing consists of 3 steps

(a) Segmentation

(b) Rotation and illumination normalization

(c) Morphological filtering.

\section{COLOR SPACE MODEL}

Color is the most important information in segmentation, gesture and object recognition and in many image processing applications. Skin colour segmentation is one of the mostly employed segmentation process in gesture recognition. Skin region detection is the primary step in gesture as well as face detection

\section{RGB COLOR SPACE MODEL}

RGB color space consists of three additive primary colors: red, green and blue. RGB color space can produce any color which can be made by the combination of these three primary colors. RGB color space works similarly as human visual system. For that reason, it is widely used color space model in Computer Vision.

$\gamma=\mathrm{R} \div(\mathrm{R}+\mathrm{G}+\mathrm{B})$

$\mathrm{g}=\mathrm{G} \div(\mathrm{R}+\mathrm{G}+\mathrm{B})$

$\mathrm{b}=\mathrm{B} \div(\mathrm{R}+\mathrm{G}+\mathrm{B})$

Here, the sum of the normalized values of the R, G and B is unity.

$$
(\mathrm{r}+\mathrm{g}+\mathrm{b})=1
$$

\section{FUTURE FRAMEWORK}

Hand gesture recognition systems can be used in many real time applications like human computer interaction (HCI), robot control, remote control, Sign language recognition etc. We have proposed a real time efficient and user independent static gesture recognition system. This work can be efficiently employed to any real time applications like VLC media player control, mouse control, remote control of a television system, robot control etc. using hand gestures.

\section{CONCLUSION}

A novel feature fusion technique for static hand gesture recognition is proposed in this work which overcomes the challenges of misclassification of geometrically closed gestures. In case of Geometric and Tchebichef moments, misclassification of gestures occurs more than Krawtchouk moment. This is because geometric moment is a local feature and it only represents the statistical attributes of the shape. On the other hand, although Tchebichef moment is orthogonal, it doesn't show satisfactory result in userindependent condition. To overcome this mismatch problem, two feature fusion strategies (serial fusion and parallel fusion) have been proposed. Experimental results show that fusion strategies significantly improve the gesture recognition performance and parallel feature fusion of Krawtchouk-Tchebichef moment has given the best gesture recognition performance.

\section{ACKNOWLEDGMENTS}

I would like to thank my supervisor Prof. Kamlesh Singh for his guidance, advice and constant support throughout my thesis work here in Ambalika Institute Of Management And Technology Lucknow.

I am highly grateful to all the faculty members and staff of the Department of Automation And Robotics, A.I.M.T Lucknow for their unforgotten teaching and motivation in my research work.

I would like to thank all my friends, lab mates .

\section{REFERENCES}

[1]. P. Kumar, J. Verma and S. Prasad, "Hand Data Glove: A Wearable real-time Device For Human-Computer Interaction" International Journal Of Advanced Science And Technology, vol. 43, Jun. 2012.

[2].https://www.nidcd.nih.gov/health/hearing/pages/asl.aspx [3]. S. P. Priyal and P. K. Bora "A Robust Static Hand Gesture Recognition System Using Geometry based Normalization and Krawtchouk Moments" Pattern Recognition, vol. 46, no. 8, pp. 2202-2219, 2013

[4]. H. Suk, S. Bong, and L. Seong. "Hand gesture recognition based on dynamic Bayesian network framework." Pattern Recognition vol. 43, no. 9, pp. 30593072, 2010. 